\title{
Disentangling Second Harmonic Generation from Multiphoton Photoluminescence in Halide Perovskites using Multidimensional Harmonic Generation
}

\author{
Darien J. Morrow, ${ }^{\dagger}$ Matthew P. Hautzinger, ${ }^{\dagger}$ David P. Lafayette $\mathrm{II},{ }^{\dagger}$ Jason M. Scheeler, ${ }^{\dagger}$ Lianna \\ Dang, ${ }^{\dagger}$ Meiying Leng, ${ }^{\dagger},+\uparrow$ Daniel D. Kohler, ${ }^{\dagger}$ Amelia M. Wheaton, ${ }^{\dagger}$ Yongping Fu, $^{\dagger}$ Ilia A. \\ Guzei, ${ }^{\dagger}$ Jiang Tang, ${ }^{\ddagger}, \uparrow$ Song Jin, ${ }^{*, \dagger}$ and John C. Wright ${ }^{*, \dagger}$ \\ $\dagger$ Department of Chemistry, University of Wisconsin-Madison, 1101 University Ave, Madison, WI \\ 53706, United States \\ $\ddagger$ Sargent Joint Research Center, Wuhan National Laboratory for Optoelectronics (WNLO), School \\ of Optical and Electronic Information, Huazhong University of Science and Technology, 1037 \\ Luoyu Road, Wuhan, 430074, Hubei, P. R. China \\ \School of Optical and Electronic Information, Huazhong University of Science and Technology, \\ 1037 Luoyu Road, Wuhan, 430074, Hubei, P. R. China \\ E-mail: jin@chem.wisc.edu; wright@chem.wisc.edu
}

Dated: June 2, 2020

\begin{abstract}
Metal halide perovskites are an intriguing class of semiconductor materials being explored for their linear and non-linear optical, and potentially ferroelectric properties. In particular, layered two-dimensional Ruddlesden-Popper (RP) halide perovskites have shown non-linear optoelectronic properties. Optical second harmonic generation (SHG) is commonly used to screen for non-centrosymmetric and ferroelectric materials, however, SHG measurements of perovskites are complicated by their intense multiphoton photoluminescence (mPL) which can be mistaken for SHG signal. In this work, we introduce multidimensional harmonic generation as a method to eliminate the complications caused by mPL. By scanning and correlating both excitation and emission frequencies, we un-ambiguously assess whether a material supports SHG by examining if an emission feature scales as twice the excitation frequency. Careful multidimensional harmonic generation measurements of a series of $n=2$ and $n=3 \mathrm{RP}$ perovskites reveal that, contrary to previous belief, $n$-butylammonium (BA) RP perovskites display no SHG, thus they have inversion symmetry; but RP perovskites with phenylethylammonium (PEA) and 2-thiophenemethylammonium (TPMA) spacer cations display SHG. Multidimensional harmonic generation is also able to confirm the SHG and thus non-centrosymmetry of a recently reported ferroelectric RP perovskite even in the presence of an obscuring $\mathrm{mPL}$ background. This work establishes multidimensional harmonic generation as a definitive method to measure the SHG properties of materials and demonstrates that tuning organic cations can allow the design of new non-centrosymmetric or even ferroelectric RP perovskites.
\end{abstract}


Two-dimensional (2D), Ruddlesden-Popper (RP) layered perovskites have emerged as an intriguing class of halide perovskites due to their quantum-well-like structure which allows for tunable optical properties and enhanced environmental stability compared to 3D lead halide perovskites, ${ }^{1-3}$ while still being easily processable into efficient solar cells. ${ }^{4,5}$ In addition, the versatile structural chemistry of RP perovskites makes them ideal candidates for applications in non-linear optoelectronics. RP perovskites have the general formula of $\left(\mathrm{RNH}_{3}\right)_{2}(\mathrm{~A})_{n-1} \mathrm{M}_{n} \mathrm{X}_{3 n+1}$ in which $\mathrm{RNH}_{3}$ is a long chain ammonium cation, including $n$-butylammonium (BA), ${ }^{6}$ phenylethylammonium (PEA), ${ }^{2,7}$ and 2-thiophenemethylammonium (TPMA); ${ }^{8,9} \mathrm{~A}$ is small, monovalent cation; $\mathrm{M}$ is a divalent metal cation (e.g. $\mathrm{Pb}^{2+}$ ); $\mathrm{X}$ is a halide anion; and $n$ is a positive integer. $\mathrm{RNH}_{3}$ acts as a spatial and dielectric barrier between the inorganic layers consisting of corner-sharing $\left[\mathrm{MX}_{6}\right]^{4-}$ octahedra, creating quantum wells that support large binding energy excitons with intense room-temperature PL. ${ }^{10}$ The value of $n$ controls the exciton binding energy and bandgap (both binding energy and bandgap decrease as $n$ increases). ${ }^{10}$ The identity of $\mathrm{M}, \mathrm{X}$ and $n$ largely determines the bandedge optical properties with the $\mathrm{A}$ and $\mathrm{RNH}_{3}$ cations having a smaller influence. ${ }^{11-13}$ However, the size and shape of the $\mathrm{RNH}_{3}$ spacer cation can impact the perovskite layers by templating the metal halide octahedral network's bonding. ${ }^{11,14,15}$ The choice of $\mathrm{RNH}_{3}$ and A cation have significant impact on the symmetry of the resulting crystal structure, and can lead to non-centrosymmetric structures, which are a prerequisite to even-order non-linear optoelectronic properties. Therefore there are many recent reports of SHG active and ferroelectric layered perovskites, including diverse $\mathrm{RP}$ perovskites like $(\mathrm{BA})_{2}(\mathrm{FA}) \mathrm{Pb}_{2} \mathrm{Br}_{7}(\mathrm{FA}=$ formamidinium $),{ }^{16}$ $(\mathrm{BA})_{2} \mathrm{CsPb}_{2} \mathrm{Br}_{7},{ }^{17}(\mathrm{BA})_{2}(\mathrm{EA})_{2} \mathrm{~Pb}_{3} \mathrm{I}_{10},{ }^{18}$ and the biaxial $(\mathrm{EA})_{4} \mathrm{~Pb}_{3} \mathrm{Br}_{10}(\mathrm{EA}=$ ethylammonium $) .{ }^{19}$ These ferroelectric materials lack inversion symmetry, exhibit an electric polarization with no applied field, and can have the direction of their polarization switched with the application of an electric field. ${ }^{20,21}$

Notably, proving that a metal halide perovskite is ferroelectric can be an arduous task. ${ }^{22-28}$ A first step involves ensuring that a candidate material lacks inversion symmetry. Optical second harmonic generation (SHG) has commonly been employed to screen ferroelectric candidates because materials that are SHG active necessarily lack inversion symmetry. ${ }^{29,30}$ Metal halide perovskites are known to efficiently support a wide variety of nonlinear optical phenomena ${ }^{31}$ including SHG in special cases, ${ }^{15,17-19,23,32,33}$ third harmonic generation (THG), ${ }^{34,35}$ high-harmonic generation up to 13th harmonic, ${ }^{36}$ and multiphoton photoluminescence (mPL). ${ }^{37,38}$ Figure 1a diagrams these phenomena. SHG and THG are spatially coherent processes with a directional output. ${ }^{30}$ SHG and THG are caused by an intense electric field driving a material polarization which then emits a new electric field with frequency components not found in the original field. The output frequencies and intensities of SHG and THG scale with the driving laser color, $\omega$, and intensity, $I$, as

$$
\begin{array}{ll}
\omega_{\mathrm{SHG}}=2 \omega & I_{\mathrm{SHG}} \propto\left|\chi^{(2)}\right|^{2} I^{2} \\
\omega_{\mathrm{THG}}=3 \omega & I_{\mathrm{THG}} \propto\left|\chi^{(3)}\right|^{2} I^{3}
\end{array}
$$

in which $\chi^{(m)}$ is the $m^{\text {th }}$-order susceptibility. Importantly, within the electric dipole approximation, if a material has inversion symmetry, then it is necessarily the case that $\chi^{(2)}=0 .{ }^{39,40}$ Thus, if a material supports SHG, the response is due either to a lack of bulk inversion symmetry, or the air-material interface which always lacks inversion symmetry. THG does not have this strict constraint and can occur in materials with inversion symmetry. These considerations imply that the presence of SHG can be a sensitive tool to probe whether a material is noncentrosymmetric. ${ }^{29}$ We consider SHG to be a complementary technique to the more definitive single-crystal X-ray 
diffraction (SCXRD) which sometimes requires additional confirmation of a crystal's symmetry group in order to determine its structure (as noted in ref. ${ }^{6}$ ).

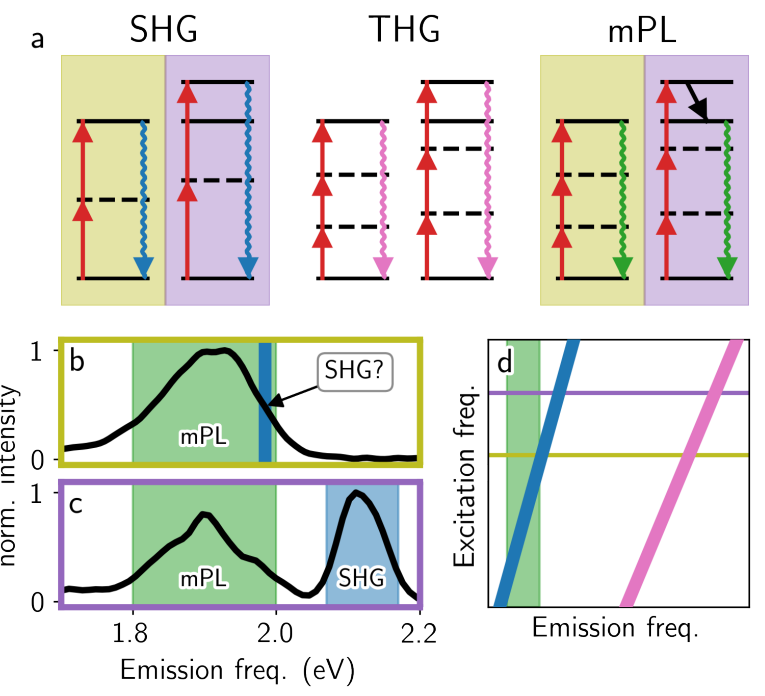

Figure 1: Comparison of various nonlinear optical processes, and methods to measure SHG. (a) energy level diagrams of SHG, THG, mPL. Arrows pointing up are the input laser, wavy arrows are emission, and diagonal arrows are cooling of hot carriers to a bandedge. Shadings are used to correlate the subfigures defining the different cases when the SHG and $\mathrm{mPL}$ have the same [yellow, see (b)] and different [lilac, see (c)] emission frequencies. (b) Measured emission spectrum from a 2D-RP perovskite when the expected SHG is within the band of $\mathrm{mPL}$ and no aperture is used to select against $\mathrm{mPL}$ - this spectrum corresponds to a conventional SHG measurement. (c) Same as (b) except the excitation laser is increased in frequency so the SHG output is moved outside the band of mPL and an aperture is used to select against mPL. (d) Diagram demonstrating how output frequency and input frequency can both be scanned to determine if a process is SHG (blue), THG (magenta), or mPL (green). Horizontal yellow and lilac lines indicate the cases shown in (b) and $(\mathrm{c})$.

However, as noted by Govinda et al. ${ }^{22}$, the strong, broad mPL present in lead halide perovskites (c.f. refs. ${ }^{37,38}$ ) can compete with weak $\mathrm{SHG}$ and lead to erroneous conclusions. In contrast to SHG and THG, mPL is caused by photons, usually with energy below the bandgap, undergoing multiphoton absorption to create hot carriers which then cool to the bandedge and isotropically fluoresce, just like carriers excited by normal, above-bandedge photon absorption. The output frequency and intensity of $\mathrm{mPL}$ will therefore be

$$
\hbar \omega_{\mathrm{mPL}} \approx E_{g} \quad I_{\mathrm{mPL}} \propto \chi^{(2 j-1)} I^{j}
$$

for $E_{g}$ being the material's optical bandgap, $j$ being the number of photons interacting in the multiphoton absorption process, and assuming no saturation effects or Stokes shift. Initially, we attempted to measure the SHG capabilities of a library of RP perovskites as part of a structureproperty study. The broad, intense mPL from the perovskites swamped our initial (conventional) SHG measurement and obscured the weak SHG signals (see Figure 1b). ${ }^{22}$

To address this challenge, here we report a multidimensional harmonic generation method which relies on a tunable excitation laser, spatial filtering, and a spectrally resolved output to ensure that 
we can definitively determine whether a material supports SHG by correlating the excitation and emission frequencies. We call this a multidimensional technique because it is analogous to coherent $2 \mathrm{D}$ spectroscopies which resolve both emission and excitation frequencies. ${ }^{41,42}$ As illustrated in Figure 1d, we can confidently assess whether a material supports SHG (or THG) because SHG (THG) will have an emission frequency that scales as twice (thrice) the excitation frequency (Equation 1, blue line, for SHG and Equation 2, magenta line, for SHG), in contrast to the $\mathrm{mPL}$ emission frequency which is not dependent on excitation frequency (Equation 3, green band). In this way, mPL emission features cannot obscure the definitive assignment of SHG features regardless of the relative intensities or peak positions of $\mathrm{mPL}$ vs. SHG. We use this technique to conclusively determine the SHG responses of RP perovskites with different $n$ values, spacer cations, and A-site cations, all of which have intense $\mathrm{mPL}$, thus elucidating and clarifying their respective crystal symmetries.

In order to access the influence of the $\mathrm{RNH}_{3}$ cation on the symmetry of the perovskite cage network, we synthesized millimeter-sized single crystals of the $n=2$ and $n=3$ variants of $\left(\mathrm{RNH}_{3}\right)_{2}(\mathrm{MA})_{n-1} \mathrm{~Pb}_{n} \mathrm{I}_{3 n+1}$ with $\mathrm{RNH}_{3}$ being one of BA, PEA, TPMA and MA being methylammonium. Figure 2 shows the crystal structures of our samples. Note that we have established the exact single-crystal structure of $(\mathrm{PEA})_{2}(\mathrm{MA}) \mathrm{Pb}_{2} \mathrm{I}_{7}$ based on the data collected from a high quality crystal at $100 \mathrm{~K}$. The synthesis details, optical micrographs (Figure S1), and powder X-ray diffraction patterns (Figure S2) confirming the phase purity of these compounds are all presented in the SI.

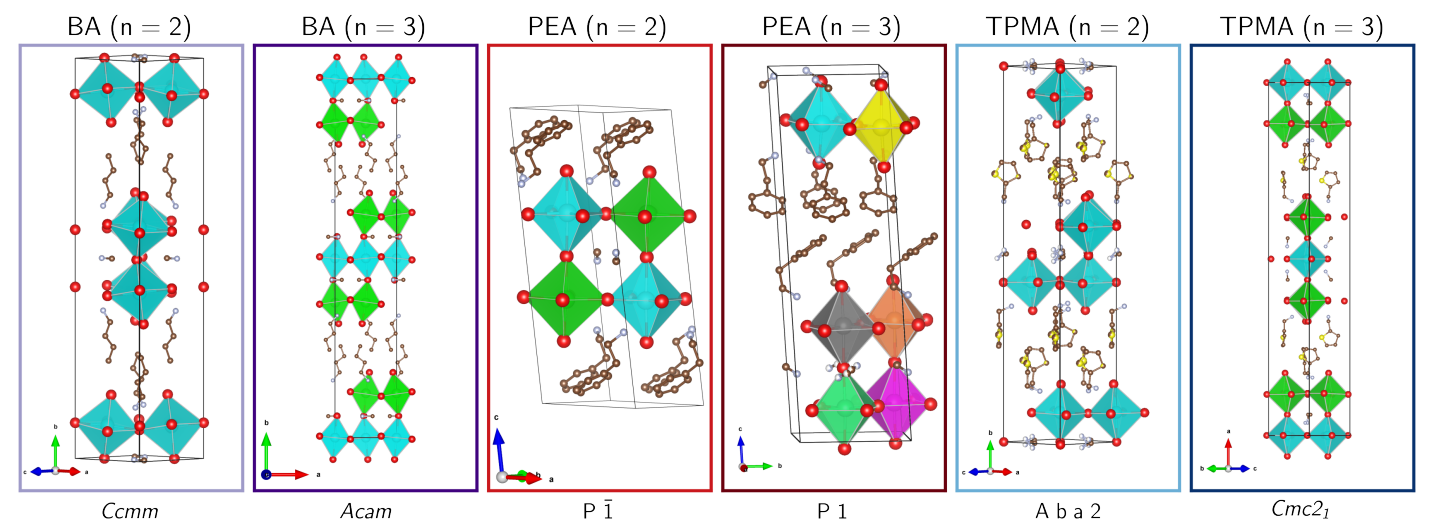

Figure 2: Crystal structures of the $n=2$ and $n=3\left(\mathrm{RNH}_{3}\right)_{2}(\mathrm{MA})_{n-1} \mathrm{~Pb}_{n} \mathrm{I}_{3 n+1}$ 2D-RP perovskites with BA, ${ }^{6} \mathrm{PEA},{ }^{2}$ and $\mathrm{TPMA}^{8,9}$ spacer cations studied in this work. The PEA $(n=2)$ structure is a new structure reported here-in. Colored octahedra are $\left[\mathrm{PbI}_{6}\right]^{4-}$, with different colors to highlight the different crystallographic sites of $\mathrm{Pb}$; for instance, in $\mathrm{BA} n=3$, all blue are $\mathrm{Pb} 1$ and green are $\mathrm{Pb} 2$ corresponding to two different symmetry-independent crystallographic sites. The color scheme for the subfigure borders used in this figure will be used consistently throughout this work.

In our measurements we use a backscattering (also known as reflective or epi) geometry to collect SHG and THG in order to minimize absorption and phase-mismatch effects. ${ }^{43,44}$ As discussed in the SI, the sampling depth SHG and THG is constrained by phase-matching to be less than $100 \mathrm{~nm}$. To perform the multidimensional harmonic generation measurements, we use an ultrafast optical parametric amplifier (OPA) to generate tunable near-IR light. This light passes through a variable neutral density filter and is loosely focused onto the sample. The SHG and THG are selected by 
an aperture, spectrally resolved with a monochromator, and detected with a photomultiplier tube. To intentionally measure PL and mPL, we remove the aperture and excite with $3.1 \mathrm{eV}$ (400 nm) or $0.99 \mathrm{eV}(1250 \mathrm{~nm})$ light, respectively; note that the collection efficiency of our photoluminescence apparatus is very low compared to a traditional PL microscope because our long focal length collection mirror leads to a numerical aperture of merely $\sim 0.05$. Additional details about our multidimensional spectrometer are described in the SI. Note that our methodology is similar to the shifted excitation difference technique used in the resonance and stimulated Raman community to remove fluorescence interference. ${ }^{45,46}$

Our main spectroscopic results are shown in Figure 3, in which we measure the output intensity for six different samples as a function of both excitation OPA frequency, $\omega$, and emission frequency, $\hbar \omega_{\mathrm{m}}$. The most consistent and prominent feature in all six spectra (right hand side) has an excitation/emission frequency dependence of $\hbar \omega_{\mathrm{m}}=3 \hbar \omega$. This feature is THG. The 2D-RP perovskites with PEA and TPMA spacer cations show a weaker but definitive feature which goes as $\hbar \omega_{\mathrm{m}}=2 \hbar \omega$. Note that this feature is SHG. Conversely, no SHG is observed for 2D-RP perovskites with BA spacer cations. Here, conclusive assignments of SHG and THG are not based merely on the presence or absence of an emission feature, but rather the scaling relationship of the emission features.

The width of the THG and SHG features along the $\hbar \omega_{\mathrm{m}}$ axis is determined by the frequency bandwidth of our ultrafast driving laser. When recording the data shown in Figure 3 we were not always able to fully reject $\mathrm{mPL}$ with our aperture. This $\mathrm{mPL}$ appears as broad features (upper left corner of each spectrum) whose emission frequency does not depend on the excitation frequency. The SHG and THG features exhibit structure which is not merely due to the non-uniform spectrum of our driving laser (Figure S3). For instance, the SHG from TPMA $n=2$ shows a sharp peak which may due to some form of excitonic enhancement. Additionally, projections of the THG spectra corrected for the excitation laser power (Figure S4) demonstrate prominent peaks between $\hbar \omega_{\mathrm{m}}=2.9-3.2 \mathrm{eV}$ - these enhancements are at significantly bluer frequencies compared to the enhancements at excitonic resonances observed by refs. ${ }^{34,35}$ in similar $2 \mathrm{D}-\mathrm{RP}$ perovskites. Because the present work is focused on the existence of SHG in materials, we elect to not provide further discussion of the structure present in the SHG and THG spectra.

Furthermore, our assignments of SHG, THG, and mPL are confirmed by measuring each feature's output intensity scaling as a function of excitation laser fluence. The data presented in Figure 4 demonstrate the expected quadratic (Equation 1) and cubic (Equation 2) output intensity scaling for SHG and THG, respectively. Slight saturation is present at the highest fluences. The $\mathrm{mPL}$ feature has cubic or higher scaling which indicates that the photoluminescence is excited by a three or more photon process.

Because SHG is a second-order process, standard heuristics dictate that it will be brighter than third-order processes such as THG. ${ }^{30,47}$ However, the observed SHG intensity in all cases herein is at least an order of magnitude less than the THG intensity (ratios tabulated in Table 1 for the specific case of $\hbar \omega=1.03 \mathrm{eV}$ ). In the SI we discuss and rule out various reasons why $I_{\mathrm{THG}} \gg I_{\mathrm{SHG}}$ and conclude that SHG is weaker than THG because the anharmonic potential felt by the optically driven electrons is only weakly noncentrosymmetric.

Figure 5 shows the single and multiphoton excited photoluminescence spectra of each sample. The marked peak positions for each sample are summarized in Table 1 . The PL spectra peak at $\sim 1.99 \mathrm{eV}(623 \mathrm{~nm})$ and $\sim 2.14 \mathrm{eV}(579 \mathrm{~nm})$ for the $n=2$ and $n=3$ variants, respectively. These values are similar to the $\sim 2.01 \mathrm{eV}$ and $\sim 2.12 \mathrm{eV}$ peaks previously reported for the $n=2$ and $n=3$ BA variants. ${ }^{6}$ All of the mPL spectra peak positions are redshifted by $\sim 0.1 \mathrm{eV}$ from the PL peak positions. A similar redshift has been reported for $3 \mathrm{D}(\mathrm{MA}) \mathrm{PbI}_{3}$ and $\mathrm{BA} 2 \mathrm{D}$ perovskites. ${ }^{35,38,49} \mathrm{We}$ 

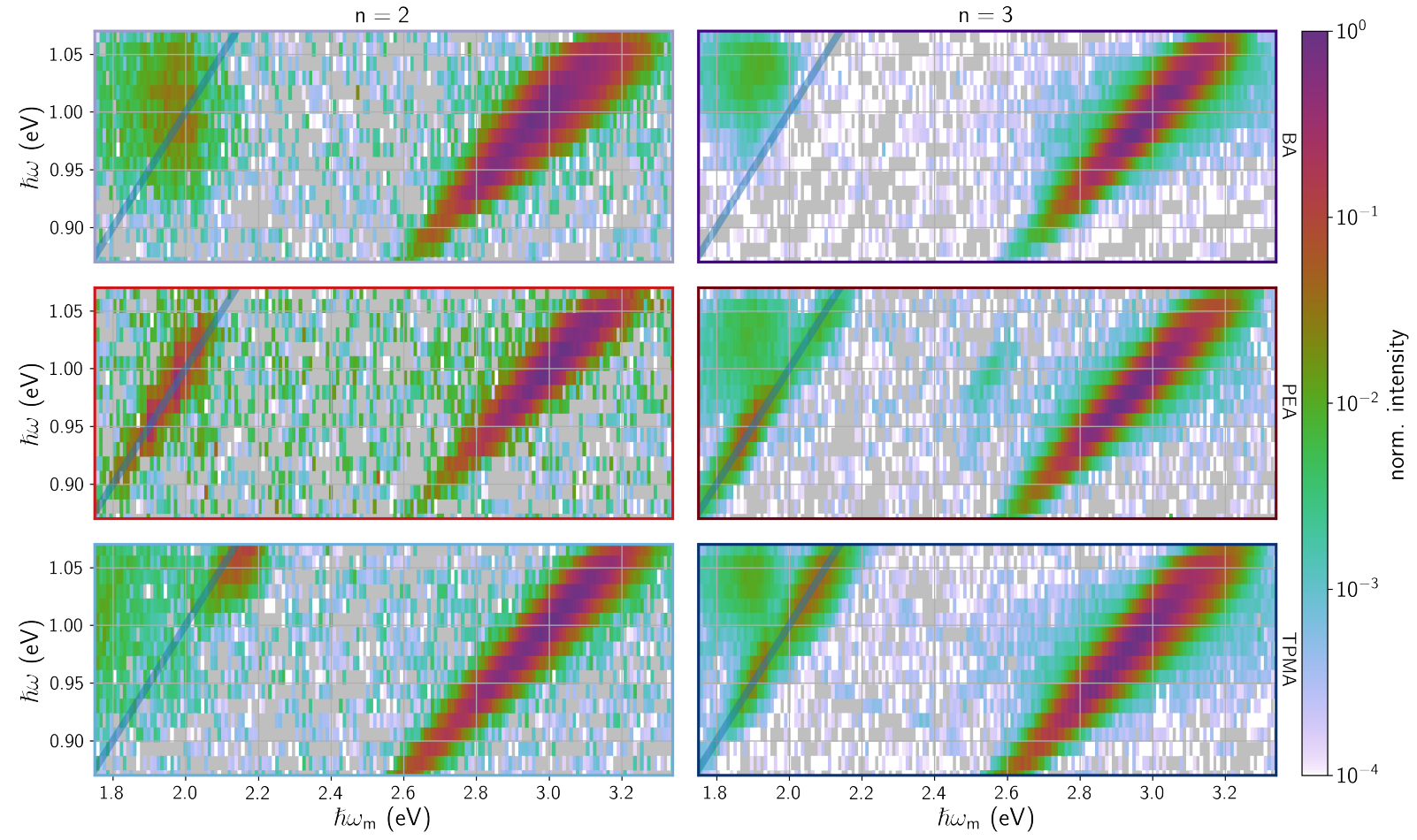

Figure 3: Multidimensional harmonic generation for six $\left(\mathrm{RNH}_{3}\right)_{2}(\mathrm{MA})_{n-1} \mathrm{~Pb}_{n} \mathrm{I}_{3 n+1}$ 2D-RP perovskite samples. $\mathrm{x}$-axis is monochromator setpoint (emission color), $\mathrm{y}$-axis is excitation laser setpoint, and colormap (in logarithmic scale, shown on the right hand side) corresponds to measured output intensity. Columns and rows correspond to $n$ and $\mathrm{RNH}_{3}$ permutations, respectively. Blue overlines indicate the SHG emission frequency. The excitation laser has a smooth variation in its spectrum which is maximized at $\hbar \omega \approx 0.96 \mathrm{eV}$ with a fluence of $\sim 1400 \mu \mathrm{J} / \mathrm{cm}^{2}$ — this variation is not corrected for in these spectra. Gray pixels indicate values which are negative and therefore unable to be represented on a logarithmic scale. These pixels characterize the noise floor of our measurement. 

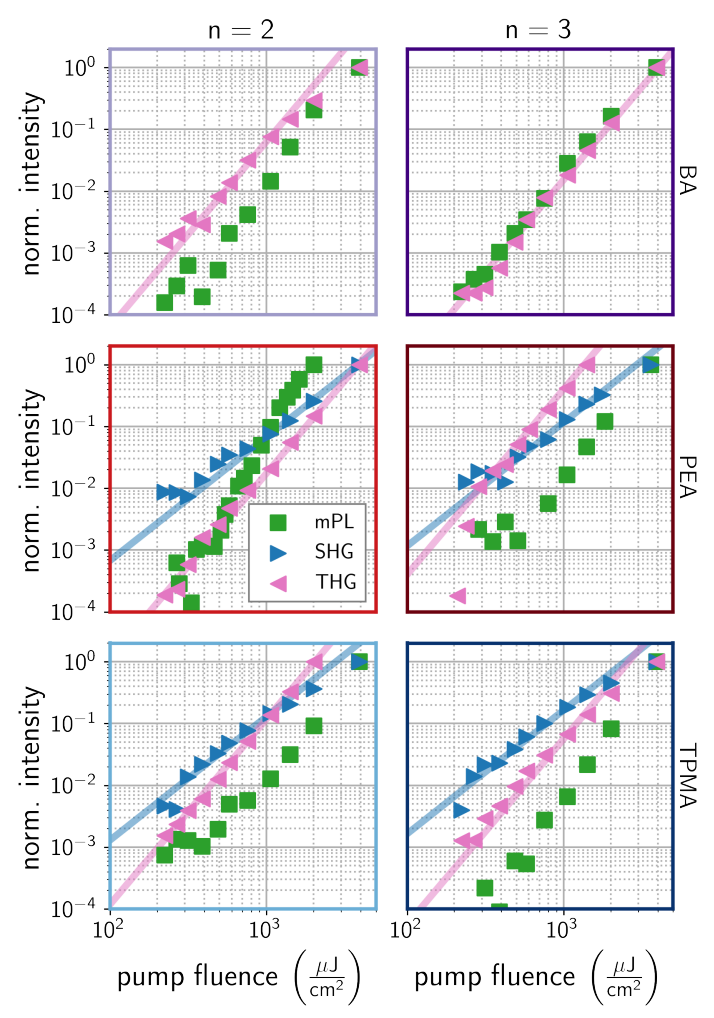

Figure 4: Scaling of the measured intensity as a function of pump fluence for SHG, THG, and mPL processes for various $\left(\mathrm{RNH}_{3}\right)_{2}(\mathrm{MA})_{n-1} \mathrm{~Pb}_{n} \mathrm{I}_{3 n+1} 2 \mathrm{D}-\mathrm{RP}$ perovskite samples. Straight lines are guides to the eye for quadratic (blue) and cubic (magenta) scaling. All measurements were performed with $\hbar \omega=0.99 \mathrm{eV}$ except for the PEA $n=2$, TPMA $n=2$, and TPMA $n=3$ SHG measurements which were performed with $\hbar \omega=1.05 \mathrm{eV}$. For SHG and THG, the monochromator was set to the second and third harmonics of the fundamental frequency, respectively, while for $\mathrm{mPL}$ the monochromator was set to the maximum of the mPL spectrum (see Figure 5 for spectra).

Table 1: Summary of the space groups and spectroscopic characterization of the six 2D-RP perovskites. $\mathrm{RNH}_{3}$ and $n$ correspond to the chemical formula of $\left(\mathrm{RNH}_{3}\right)_{2}(\mathrm{MA})_{n-1} \mathrm{~Pb}_{n} \mathrm{I}_{3 n+1}$. The peak values for photoluminescence correspond to the vertical bars shown in Figure $5 . I_{\mathrm{SHG}} / I_{\mathrm{THG}}$ was determined from the data shown in Figure 3 for $\hbar \omega=1.03(\mathrm{eV})$ with a fluence of $\sim 1400 \mu \mathrm{J} / \mathrm{cm}^{2}$. Note, that without considering phase-matching, absorption, and instrument response effects, comparison by a ratio of intensities is problematic. However, we report ratios here in order to compare to past literature (e.g. ref. $^{6}$ ), and because we are comparing materials with similar bandgaps at identical emission frequencies these ratios offer a fairly self-consistent comparison.

\begin{tabular}{|c|c|c|c|c|c|c|}
\hline $\mathrm{RNH}_{3}$ & $n$ & space group & mPL peak $(\mathrm{eV})$ & PL peak $(\mathrm{eV})$ & $I_{\mathrm{SHG}} / I_{\mathrm{THG}}$ & SHG? \\
\hline $\mathrm{BA}$ & 2 & orthorhombic $C c 2 m$ (at 293(2) K) (No. 40) Or $C c m m$. Ref. ${ }^{6}$ & 1.98 & 2.12 & $<0.001$ & no \\
\hline BA & 3 & orthorhombic $C 2 c b$ (at 293(2) K) (No. 41) Or Acam. Ref. ${ }^{6}$ & 1.875 & 2.00 & $<0.001$ & no \\
\hline PEA & 2 & P $\overline{1}$ (No. 2). Ref. ${ }^{48}$ and this work & 2.05 & 2.15 & 0.10 & yes \\
\hline PEA & 3 & P 1 (No. 1). Ref. ${ }^{2}$ & 1.91 & 1.98 & 0.02 & yes \\
\hline TPMA & 2 & orthorhombic A b a 2 (293 K) (No. 41). Ref. $^{8}$ & 2.00 & 2.14 & 0.02 & yes \\
\hline TPMA & 3 & orthorhombic, $C m c 2_{1}$ (No. 36$) 100 \mathrm{~K}$ and $296 \mathrm{~K}$ (no phase change). Ref. ${ }^{9}$ & 1.90 & 1.995 & 0.10 & yes \\
\hline
\end{tabular}


attribute the redshift to a photon recycling effect. ${ }^{38,49}$ Multiphoton absorption can create excited carriers much deeper into a sample than single photon absorption. The deeper that carriers are excited in a sample, the more likely their photoluminescence will be reabsorbed to create other carriers. The Stokes-shift associated with the photoluminescence will lead to the reddest photons selectively being able to exit the sample.
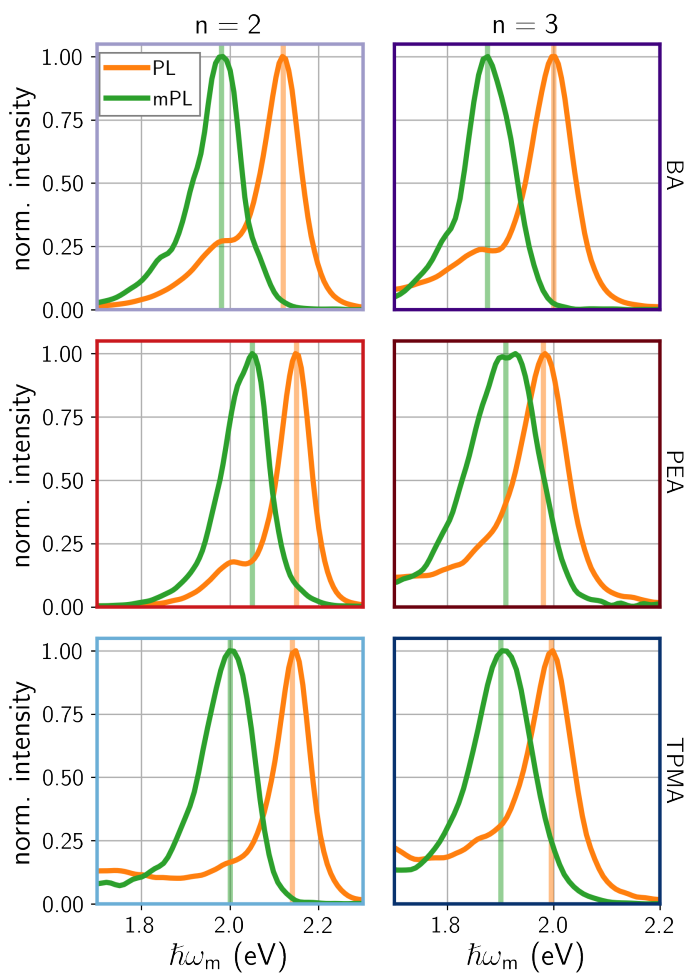

Figure 5: Normalized PL (3.1 eV excitation) and $\mathrm{mPL}$ (0.99 eV excitation) spectra for various $\left(\mathrm{RNH}_{3}\right)_{2}(\mathrm{MA})_{n-1} \mathrm{~Pb}_{n} \mathrm{I}_{3 n+1} 2 \mathrm{D}-\mathrm{RP}$ perovskite samples. Vertical lines correspond to approximate peak maxima and are recorded in Table 1.

The presence of SHG in RP perovskites made with PEA and TPMA spacer cations but not BA cations indicates that the bulky PEA and TPMA cations template different bonding in the inorganic lead iodide network. Single-crystal X-ray diffraction structures show that the TPMA $n=2$, TPMA $n=3$, and PEA $n=32 \mathrm{D}$-RP perovskites have noncentrosymmetric space groups (see Table 1). ${ }^{2,8,9}$ The previously reported SCXRD structure refinements of BA $n=2$ and $n=3$ 2D-RP perovskites showed that they could either be centrosymmetric or noncentrosymmetric space groups, but a weak SHG response, $I_{\mathrm{SHG}} / I_{\mathrm{THG}} \approx 0.01$, was measured with a single excitation color which was used as the basis to conclude that BA $n=2$ and $n=3$ are noncentrosymmetric. ${ }^{6}$ Our multidimensional harmonic generation experiments did not reveal SHG in any of the BA $n=2$ and $n=3$ crystals we examined. We further quantify the limit of our ability to measure SHG (Figure S5) and find that our detection limit is three orders of magnitude lower than the limit needed to observe SHG at the previously reported SHG to THG ratio. ${ }^{6}$ It is possible that local symmetry-breaking effects were at play which led to some weak SHG signal, ${ }^{6}$ as noted by the authors. It is also possible that mPL photons from low energy "edge" states ${ }^{50,51}$ were measured 
and attributed to SHG. The lack of SHG in our multidimensional measurements on BA $n=2$ and $n=3$ crystals indicates that these crystal structures are better described as centrosymmetric in the $C \mathrm{cmm}$ and Acam space groups, respectively, instead of the previously assigned space groups of $C c 2 m$ and $C 2 c b .{ }^{6}$ The main difference between the centrosymmetric and non-centrosymmetric structures is whether the methylammonium cations are disordered or ordered, respectively. ${ }^{6}$

Based on previous SCXRD measurements, the PEA $n=2$ structure is believed to be centrosymmetric. ${ }^{48}$ However, this crystal structure (originally reported in 1991) was based on a partial structural model with incomplete organic cation atom positions, likely due to their high degree of disorder at the $298 \mathrm{~K}$ experiment temperature. ${ }^{48}$ We therefore grew high-quality single crystals (see Figure S1) of the PEA $n=2$ phase and carried out SCXRD analysis at $100 \mathrm{~K}$. Our complete structural refinement yielded a centrosymmetric space group with a well-defined PEA cation disorder. A structural refinement was also attempted in a non-centrosymmetric group, however the same PEA cation disorder was observed and numerical indicators suggested a higher symmetry (centrosymmetric) space group. Since our structural refinement confirms the centrosymmetry of PEA $n=2$, it is tempting to attribute the observed SHG from PEA $n=2$ to surface effects or localized symmetry breaking domains. To investigate this contradiction between observing SHG and a centrosymmetric structure from SCXRD, we examined many PEA $n=2$ crystals from different synthesis batches and observed SHG in all of them (data shown in Figure S6 and Figure S7). Some samples displayed a greater SHG to THG ratio than others, indicating inhomogeneity among the crystals. In an attempt to mitigate local defects, we also grew PEA $n=2$ using an alternative, slow, organic solvent based growth method. ${ }^{2}$ The resultant crystals also supported SHG. We intentionally degraded PEA $n=2$ crystals in a humidity chamber and observed significantly more $\mathrm{mPL}$ from the $n=2$ phase and the emergence of $\mathrm{mPL}$ from the $n=1$ phase that was formed upon degradation. Crucially, we did not observe a significant increase in SHG efficiency when a sample had its surface degraded. This observation suggests that surface effects are not the dominant contributor to such observed SHG.

Tentatively, PEA $n=2$ samples display at least local noncentrosymmetry, which may be due to the disorder in the cations contributing to a noncentrosymmetric structure not observable in SCXRD measurements. The possibility of localized and spatially dependent SHG response could be investigated by future microscopy studies. ${ }^{52}$ Other researchers have found the disordered nature of PEA $n=1$ cations to be an important influence to breaking local degeneracy in the excitonic energy landscape of thin films. ${ }^{53-55}$ Their observations, like ours, suggest the importance of $\mathrm{RNH}_{3}$ cations in determining the symmetry of the electronic states of 2D-RP perovskites. Our multidimensional harmonic generation method could be extended in the future to measuring excited state symmetries (like those considered in refs. ${ }^{53-55}$ ) by the addition of an optical pump. ${ }^{56,57}$

To further demonstrate the capability of multidimensional harmonic generation to discriminate against $\mathrm{mPL}$, we grew a single crystal of $n=3(\mathrm{BA})_{2}(\mathrm{EA})_{2} \mathrm{~Pb}_{3} \mathrm{I}_{10}$, which has recently been identified as a 2D-RP perovskite that is ferroelectric at room temperature. ${ }^{18}$ As shown in Figure 6, this ferroelectric 2D-RP perovskite has intense $\mathrm{mPL}$ that could obfuscate a conventional SHG measurement. However, because we observe an unambiguous feature (highlighted by a blue line) whose output frequency tracks twice the input frequency, we can be assured that $(\mathrm{BA})_{2}(\mathrm{EA})_{2} \mathrm{~Pb}_{3} \mathrm{I}_{10}$ is indeed SHG active, and thus non-centrosymmetric, and therefore possibly a ferroelectric material at room temperature. The confirmation that a $2 \mathrm{D}-\mathrm{RP}$ perovskite with a butylammonium spacer cation and ethylammonium A-site cation is non-centrosymmetric is especially of note because the analogous $(\mathrm{BA})_{2}(\mathrm{MA})_{2} \mathrm{~Pb}_{3} \mathrm{I}_{10} \mathrm{RP}$ perovskites with MA in the perovskite cage have been confirmed to be centrosymmetric (see results and discussion above). This observation indicates that small permutations in both the A-site and $\mathrm{RNH}_{3}$ spacer cations can influence the inversion symmetry of 
the entire 2D-RP perovskite crystal structure. ${ }^{15}$

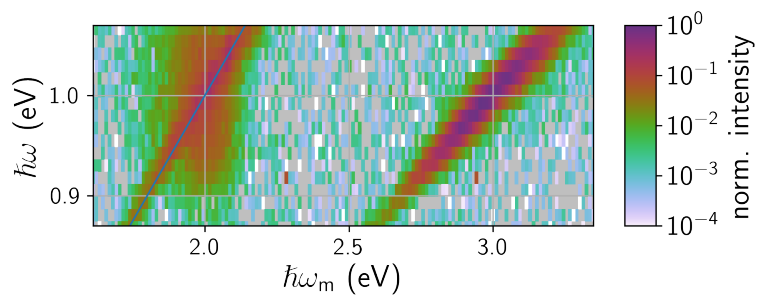

Figure 6: Multidimensional harmonic generation of a $(\mathrm{BA})_{2}(\mathrm{EA})_{2} \mathrm{~Pb}_{3} \mathrm{I}_{10}$ single crystal. This compound is reported by Han et al. ${ }^{18}$ to be ferroelectric at room temperature. The blue overline indicates the SHG emission frequency.

We have shown that multidimensional harmonic generation can be used to definitively measure and clarify the SHG and THG response of halide perovskite materials, even in the presence of a strong mPL background. By scanning and correlating both excitation and emission frequencies, we unambiguously assess whether a material supports SHG. This methodology is systematically applied to single crystals of seven $2 \mathrm{D}-\mathrm{RP}$ perovskites with different spacer cations, $n$ values, and A cations. The lack of SHG in $n=2$ and $n=32 \mathrm{D}-\mathrm{RP}$ perovskites with BA spacer cations definitively indicates that these materials have a centrosymmetric crystal structure instead of the previously reported non-centrosymmetric structures based on inconclusive SHG measurements. Observation of SHG from $n=2$ and $n=3$ 2D-RP perovskites with PEA and TPMA spacer cations demonstrate that bulky, aromatic spacer cations can template the lead iodide network creating materials that lack inversion symmetry. There remains a contradiction between our SCXRD analysis that shows the $(\mathrm{PEA})_{2}(\mathrm{MA}) \mathrm{Pb}_{2} \mathrm{I}_{7}$ crystal is centrosymmetric, and the observation of SHG. This contradiction should be investigated by future microscopy studies. ${ }^{52}$ We further showed that multidimensional harmonic generation confirms the noncentrosymmetry of the recently reported ferroelectric 2D$\mathrm{RP}$ perovskite $(\mathrm{BA})_{2}(\mathrm{EA})_{2} \mathrm{~Pb}_{3} \mathrm{I}_{10}$ even in the presence of bright $\mathrm{mPL} .{ }^{18}$ This work establishes multidimensional harmonic generation as a definitive technique to reveal the SHG properties of a material. Our results also demonstrate that subtle variations in A-site and $\mathrm{RNH}_{3}$ spacer cations can be used to control the overall crystal symmetry of $2 \mathrm{D}$-RP perovskites as strategies to engineer and design next generation ferroelectric materials.

\section{Acknowledgement}

This work is supported by the Department of Energy, Office of Basic Energy Sciences, Division of Materials Sciences and Engineering, under award DE-FG02-09ER46664. D.J.M. acknowledges support from the Link Foundation. L. D. acknowledges the support of an NSF Graduate Research Fellowship (DGE-1747503) as well as the Graduate School and the Office of the Vice Chancellor for Research and Graduate Education at the University of Wisconsin-Madison with funding from the Wisconsin Alumni Research Foundation. M. L. acknowledges fellowship support from the China Scholarship Council. M. L. and J. T. acknowledge support from the National Natural Science Foundation of China (51761145048). 


\section{Supporting Information Available}

Additional supporting information for this work including sample synthesis details, PXRD patterns, and micrographs, details about the multidimensional harmonic generation spectrometer, SCXRD details, crystal data for compound $(\mathrm{PEA})_{2}(\mathrm{MA}) \mathrm{Pb}_{2} \mathrm{I}_{7}$ which is also available via the CCDC at www . ccdc.cam.ac.uk/data_request/cif with accession code 1978042, a quantification of our inability to observe SHG in BA $n=3$, harmonic generation data on many PEA $n=2$ samples, and a derivation/discussion concerning how the degree of non-centrosymmetry of an anharmonic restoring potential controls the relative intensity of SHG and THG. The materials are available free of charge via the journal website publisher should insert URL. All data and the workup/representation scripts used to generate the figures in this work are permissively licensed and publicly available for reuse at DOI: 10.17605/OSF. IO/JN24U. 


\section{References}

(1) Mao, L.; Stoumpos, C. C.; Kanatzidis, M. G. Two-Dimensional Hybrid Halide Perovskites: Principles and Promises. J. Am. Chem. Soc. 2018, 141, 1171-1190, DOI: 10.1021/jacs. $8 \mathrm{~b} 10851$.

(2) Smith, I. C.; Hoke, E. T.; Solis-Ibarra, D.; McGehee, M. D.; Karunadasa, H. I. A Layered Hybrid Perovskite Solar-Cell Absorber with Enhanced Moisture Stability. Angew. Chem 2014, 53, 11232-11235, DOI: 10.1002/anie.201406466.

(3) Fu, Y.; Zhu, H.; Chen, J.; Hautzinger, M. P.; Zhu, X.-Y.; Jin, S. Metal halide perovskite nanostructures for optoelectronic applications and the study of physical properties. Nat. Rev. Mater. 2019, 4, 169-188, DOI: 10.1038/s41578-019-0080-9.

(4) Tsai, H. et al. High-efficiency two-dimensional Ruddlesden-Popper perovskite solar cells. $\mathrm{Na}$ ture 2016, 536, 312-316, DOI: 10.1038/nature18306.

(5) Cao, D. H.; Stoumpos, C. C.; Farha, O. K.; Hupp, J. T.; Kanatzidis, M. G. 2D Homologous Perovskites as Light-Absorbing Materials for Solar Cell Applications. J. Am. Chem. Soc. 2015, 137, 7843-7850, DOI: 10.1021/jacs.5b03796.

(6) Stoumpos, C. C.; Cao, D. H.; Clark, D. J.; Young, J.; Rondinelli, J. M.; Jang, J. I.; Hupp, J. T.; Kanatzidis, M. G. Ruddlesden-Popper Hybrid Lead Iodide Perovskite 2D Homologous Semiconductors. Chem. Mater. 2016, 28, 2852-2867, DOI: 10.1021/acs.chemmater.6b00847.

(7) Ma, D.; Fu, Y.; Dang, L.; Zhai, J.; Guzei, I. A.; Jin, S. Single-crystal microplates of twodimensional organic-inorganic lead halide layered perovskites for optoelectronics. Nano Res. 2017, 10, 2117-2129, DOI: 10.1007/s12274-016-1401-6.

(8) Zhu, X.-H.; Mercier, N.; Riou, A.; Blanchard, P.; Frère, P. $\left(\mathrm{C}_{4} \mathrm{H}_{3} \mathrm{SCH}_{2} \mathrm{NH}_{3}\right)_{2}\left(\mathrm{CH}_{3} \mathrm{NH}_{3}\right) \mathrm{Pb}_{2} \mathrm{I}_{7}$ : non-centrosymmetrical crystal structure of a bilayer hybrid perovskite. Chem. Commun. 2002, 2160-2161, DOI: 10.1039/b205543k.

(9) Lai, H.; Kan, B.; Liu, T.; Zheng, N.; Xie, Z.; Zhou, T.; Wan, X.; Zhang, X.; Liu, Y.; Chen, Y. Two-Dimensional Ruddlesden-Popper Perovskite with Nanorod-like Morphology for Solar Cells with Efficiency Exceeding 15\%. J. Am. Chem. Soc. 2018, 140, 11639-11646, DOI: $10.1021 /$ jacs.8b04604.

(10) Blancon, J.-C. et al. Scaling law for excitons in 2D perovskite quantum wells. Nat. Commun. 2018, 9, DOI: $10.1038 / \mathrm{s} 41467-018-04659-\mathrm{x}$.

(11) Du, K.; Tu, Q.; Zhang, X.; Han, Q.; Liu, J.; Zauscher, S.; Mitzi, D. B. Two-Dimensional Lead(II) Halide-Based Hybrid Perovskites Templated by Acene Alkylamines: Crystal Structures, Optical Properties, and Piezoelectricity. Inorg. Chem. 2017, 56, 9291-9302, DOI: 10.1021/acs. inorgchem.7b01094.

(12) Fu, Y.; Hautzinger, M. P.; Luo, Z.; Wang, F.; Pan, D.; Aristov, M. M.; Guzei, I. A.; Pan, A.; Zhu, X.; Jin, S. Incorporating Large A Cations into Lead Iodide Perovskite Cages: Relaxed Goldschmidt Tolerance Factor and Impact on Exciton-Phonon Interaction. ACS Cent. Sci. 2019, 5, 1377-1386, DOI: 10.1021/acscentsci.9b00367. 
(13) Hautzinger, M. P.; Pan, D.; Pigg, A. K.; Fu, Y.; Morrow, D. J.; Leng, M.; Kuo, M.-Y.; Spitha, N.; Lafayette, D. P.; Kohler, D. D.; Wright, J. C.; Jin, S. Band Edge Tuning of TwoDimensional Ruddlesden-Popper Perovskites by A Cation Size Revealed through Nanoplates. ACS Energy Lett. 2020, 5, 1430-1437, DOI: 10.1021/acsenergylett.0c00450.

(14) Saparov, B.; Mitzi, D. B. Organic-Inorganic Perovskites: Structural Versatility for Functional Materials Design. Chem. Rev. 2016, 116, 4558-4596, DOI: 10.1021/acs.chemrev.5b00715.

(15) Schmitt, T.; Bourelle, S.; Tye, N.; Soavi, G.; Bond, A. D.; Feldmann, S.; Traore, B.; Katan, C.; Even, J.; Dutton, S. E.; Deschler, F. Control of Crystal Symmetry Breaking with HalogenSubstituted Benzylammonium in Layered Hybrid Metal-Halide Perovskites. J. Am. Chem. Soc. 2020, DOI: $10.1021 /$ jacs.9b11809.

(16) Li, L.; Shang, X.; Wang, S.; Dong, N.; Ji, C.; Chen, X.; Zhao, S.; Wang, J.; Sun, Z.; Hong, M.; Luo, J. Bilayered Hybrid Perovskite Ferroelectric with Giant Two-Photon Absorption. J. Am. Chem. Soc. 2018, 140, 6806-6809, DOI: 10.1021/jacs.8b04014.

(17) Wu, Z.; Ji, C.; Li, L.; Kong, J.; Sun, Z.; Zhao, S.; Wang, S.; Hong, M.; Luo, J. Alloying n -Butylamine into $\mathrm{CsPbBr}_{3}$ To Give a Two-Dimensional Bilayered Perovskite Ferroelectric Material. Angew. Chem. 2018, 57, 8140-8143, DOI: 10.1002/anie. 201803716.

(18) Han, S.; Liu, X.; Liu, Y.; Xu, Z.; Li, Y.; Hong, M.; Luo, J.; Sun, Z. High-Temperature Antiferroelectric of Lead Iodide Hybrid Perovskites. J. Am. Chem. Soc. 2019, 141, 1247012474, DOI: $10.1021 /$ jacs.9b05124.

(19) Wang, S.; Liu, X.; Li, L.; Ji, C.; Sun, Z.; Wu, Z.; Hong, M.; Luo, J. An Unprecedented Biaxial Trilayered Hybrid Perovskite Ferroelectric with Directionally Tunable Photovoltaic Effects. J. Am. Chem. Soc. 2019, 141, 7693-7697, DOI: 10.1021/jacs.9b02558.

(20) Butler, K. T.; Frost, J. M.; Walsh, A. Ferroelectric materials for solar energy conversion: photoferroics revisited. Energy Environ. Sci. 2015, 8, 838-848, DOI: 10.1039/c4ee03523b.

(21) Paillard, C.; Bai, X.; Infante, I. C.; Guennou, M.; Geneste, G.; Alexe, M.; Kreisel, J.; Dkhil, B. Photovoltaics with Ferroelectrics: Current Status and Beyond. Adv. Mater. 2016, 28, 51535168, DOI: 10.1002/adma. 201505215.

(22) Govinda, S.; Kore, B. P.; Mahale, P.; Pandey, A.; Sarma, D. D. Can SHG Measurements Determine the Polarity of Hybrid Lead Halide Perovskites? ACS Energy Lett. 2018, 3, 18871891, DOI: 10.1021/acsenergylett.8b00999.

(23) Rakita, Y.; Bar-Elli, O.; Meirzadeh, E.; Kaslasi, H.; Peleg, Y.; Hodes, G.; Lubomirsky, I.; Oron, D.; Ehre, D.; Cahen, D. Tetragonal $\mathrm{CH}_{3} \mathrm{NH}_{3} \mathrm{PbI}_{3}$ is ferroelectric. Proc. Natl. Acad. Sci. U.S.A. 2017, 114, E5504-E5512, DOI: 10.1073/pnas.1702429114.

(24) Röhm, H.; Leonhard, T.; Schulz, A. D.; Wagner, S.; Hoffmann, M. J.; Colsmann, A. Ferroelectric Properties of Perovskite Thin Films and Their Implications for Solar Energy Conversion. Adv. Mater. 2019, 31, 1806661, DOI: 10.1002/adma. 201806661.

(25) Fan, Z.; Xiao, J.; Sun, K.; Chen, L.; Hu, Y.; Ouyang, J.; Ong, K. P.; Zeng, K.; Wang, J. Ferroelectricity of $\mathrm{CH}_{3} \mathrm{NH}_{3} \mathrm{PbI}_{3}$ Perovskite. J. Phys. Chem. Lett. 2015, 6, 1155-1161, DOI: 10.1021/acs.jpclett.5b00389. 
(26) G, S.; Mahale, P.; Kore, B. P.; Mukherjee, S.; Pavan, M. S.; De, C.; Ghara, S.; Sundaresan, A.; Pandey, A.; Row, T. N. G.; Sarma, D. D. Is $\mathrm{CH}_{3} \mathrm{NH}_{3} \mathrm{PbI}_{3}$ Polar? J. Phys. Chem. Lett. 2016, 7, 2412-2419, DOI: 10.1021/acs.jpclett.6b00803.

(27) Gao, Z.-R.; Sun, X.-F.; Wu, Y.-Y.; Wu, Y.-Z.; Cai, H.-L.; Wu, X. S. Ferroelectricity of the Orthorhombic and Tetragonal MAPbBr3 Single Crystal. J. Phys. Chem. Lett. 2019, 10, 25222527, DOI: 10.1021/acs.jpclett.9b00776.

(28) Beilsten-Edmands, J.; Eperon, G. E.; Johnson, R. D.; Snaith, H. J.; Radaelli, P. G. Nonferroelectric nature of the conductance hysteresis in $\mathrm{CH}_{3} \mathrm{NH}_{3} \mathrm{PbI}_{3}$ perovskite-based photovoltaic devices. Appl. Phys. Lett. 2015, 106, 173502, DOI: 10.1063/1.4919109.

(29) Ok, K. M.; Chi, E. O.; Halasyamani, P. S. Bulk characterization methods for noncentrosymmetric materials: second-harmonic generation, piezoelectricity, pyroelectricity, and ferroelectricity. Chem. Soc. Rev. 2006, 35, 710, DOI: 10.1039/b511119f.

(30) Boyd, R. W. Nonlinear Optics, 3rd ed.; Academic Press, 2008.

(31) Xu, J.; Li, X.; Xiong, J.; Yuan, C.; Semin, S.; Rasing, T.; Bu, X.-H. Halide Perovskites for Nonlinear Optics. Adv. Mater. 2019, 1806736, DOI: 10.1002/adma. 201806736.

(32) Liao, W.-Q.; Zhang, Y.; Hu, C.-L.; Mao, J.-G.; Ye, H.-Y.; Li, P.-F.; Huang, S. D.; Xiong, R.G. A lead-halide perovskite molecular ferroelectric semiconductor. Nat. Commun. 2015, 6, DOI: $10.1038 /$ ncomms8338.

(33) Wei, W.-J.; Jiang, X.-X.; Dong, L.-Y.; Liu, W.-W.; Han, X.-B.; Qin, Y.; Li, K.; Li, W.; Lin, Z.-S.; Bu, X.-H.; Lu, P.-X. Regulating Second-Harmonic Generation by van der Waals Interactions in Two-dimensional Lead Halide Perovskite Nanosheets. J. Am. Chem. Soc. 2019, 141, 9134-9139, DOI: 10.1021/jacs.9b01874.

(34) Abdelwahab, I.; Grinblat, G.; Leng, K.; Li, Y.; Chi, X.; Rusydi, A.; Maier, S. A.; Loh, K. P. Highly Enhanced Third-Harmonic Generation in 2D Perovskites at Excitonic Resonances. ACS Nano 2017, 12, 644-650, DOI: 10.1021/acsnano.7b07698.

(35) Saouma, F. O.; Stoumpos, C. C.; Wong, J.; Kanatzidis, M. G.; Jang, J. I. Selective enhancement of optical nonlinearity in two-dimensional organic-inorganic lead iodide perovskites. Nat. Commun. 2017, 8, DOI: 10.1038/s41467-017-00788-x.

(36) Hirori, H.; Xia, P.; Shinohara, Y.; Otobe, T.; Sanari, Y.; Tahara, H.; Ishii, N.; Itatani, J.; Ishikawa, K. L.; Aharen, T.; Ozaki, M.; Wakamiya, A.; Kanemitsu, Y. High-order harmonic generation from hybrid organic-inorganic perovskite thin films. APL Mater. 2019, 7, 041107, DOI: $10.1063 / 1.5090935$.

(37) Clark, D. J.; Stoumpos, C. C.; Saouma, F. O.; Kanatzidis, M. G.; Jang, J. I. Polarizationselective three-photon absorption and subsequent photoluminescence inCsPbBr3single crystal at room temperature. Phys. Rev. B 2016, 93, DOI: 10.1103/physrevb.93.195202.

(38) Gan, Z.; Wen, X.; Chen, W.; Zhou, C.; Yang, S.; Cao, G.; Ghiggino, K. P.; Zhang, H.; Jia, B. The Dominant Energy Transport Pathway in Halide Perovskites: Photon Recycling or Carrier Diffusion? Adv. Energy Mater. 2019, 9, 1900185, DOI: 10.1002/aenm. 201900185. 
(39) Shen, Y. R. The Principles of Nonlinear Optics, 1st ed.; John Wiley \& Sons, 1984.

(40) Bloembergen, N.; Chang, R. K.; Jha, S. S.; Lee, C. H. Optical Second-Harmonic Generation in Reflection from Media with Inversion Symmetry. Phys. Rev. 1968, 174, 813-822, DOI: 10.1103/physrev.174.813.

(41) Gelzinis, A.; Augulis, R.; Butkus, V.; Robert, B.; Valkunas, L. Two-dimensional spectroscopy for non-specialists. Biochim. Biophys. Acta 2019, 1860, 271-285, DOI: 10.1016/j.bbabio. 2018.12 .006$.

(42) Wright, J. C. Multiresonant Coherent Multidimensional Spectroscopy. Annu. Rev. Phys. Chem. 2011, 62, 209-230, DOI: 10.1146/annurev-physchem-032210-103551.

(43) Morrow, D. J.; Kohler, D. D.; Wright, J. C. Group- and phase-velocity-mismatch fringes in triple sum-frequency spectroscopy. Phys. Rev. A 2017, 96, 063835, DOI: 10.1103/physreva. 96.063835 .

(44) Morrow, D. J.; Kohler, D. D.; Czech, K. J.; Wright, J. C. Communication: Multidimensional triple sum-frequency spectroscopy of $\mathrm{MoS}_{2}$ and comparisons with absorption and second harmonic generation spectroscopies. J. Chem. Phys. 2018, 149, 091101, DOI: $10.1063 / 1.5047802$.

(45) Shreve, A. P.; Cherepy, N. J.; Mathies, R. A. Effective Rejection of Fluorescence Interference in Raman Spectroscopy Using a Shifted Excitation Difference Technique. Appl. Spectrosc. 1992, 46, 707-711, DOI: 10.1366/0003702924125122.

(46) Bera, K.; Kwang, S. Y.; Cassabaum, A. A.; Rich, C. C.; Frontiera, R. R. Facile Background Discrimination in Femtosecond Stimulated Raman Spectroscopy Using a Dual-Frequency Raman Pump Technique. J. Phys. Chem. A 2019, 123, 7932-7939, DOI: 10.1021/acs.jpca. 9b02473.

(47) Boyd, R. W. Order-of-magnitude estimates of the nonlinear optical susceptibility. J. Mod. Opt. 1999, 46, 367-378, DOI: 10.1080/09500349908231277.

(48) Calabrese, J.; Jones, N. L.; Harlow, R. L.; Herron, N.; Thorn, D. L.; Wang, Y. Preparation and characterization of layered lead halide compounds. J. Am. Chem. Soc. 1991, 113, 2328-2330, DOI: $10.1021 / \mathrm{ja} 00006 \mathrm{a} 076$.

(49) Yamada, Y.; Yamada, T.; Phuong, L. Q.; Maruyama, N.; Nishimura, H.; Wakamiya, A.; Murata, Y.; Kanemitsu, Y. Dynamic Optical Properties of $\mathrm{CH}_{3} \mathrm{NH}_{3} \mathrm{PbI}_{3}$ Single Crystals As Revealed by One- and Two-Photon Excited Photoluminescence Measurements. J. Am. Chem. Soc. 2015, 137, 10456-10459, DOI: 10.1021/jacs.5b04503.

(50) Blancon, J.-C. et al. Extremely efficient internal exciton dissociation through edge states in layered 2D perovskites. Science 2017, 355, 1288-1292, DOI: 10.1126/science.aal4211.

(51) Shi, E.; Deng, S.; Yuan, B.; Gao, Y.; Akriti,; Yuan, L.; Davis, C. S.; Zemlyanov, D.; Yu, Y.; Huang, L.; Dou, L. Extrinsic and Dynamic Edge States of Two-Dimensional Lead Halide Perovskites. ACS Nano 2019, DOI: 10.1021/acsnano.8b07631. 
(52) Jung, H. J.; Stompus, C. C.; Kanatzidis, M. G.; Dravid, V. P. Self-Passivation of 2D Ruddlesden-Popper Perovskite by Polytypic Surface $\mathrm{PbI}_{2}$ Encapsulation. Nano Lett. 2019, 19, 6109-6117, DOI: 10.1021/acs.nanolett.9b02069.

(53) Thouin, F.; Valverde-Chávez, D. A.; Quarti, C.; Cortecchia, D.; Bargigia, I.; Beljonne, D.; Petrozza, A.; Silva, C.; Kandada, A. R. S. Phonon coherences reveal the polaronic character of excitons in two-dimensional lead halide perovskites. Nat. Mater. 2019, 18, 349-356, DOI: $10.1038 / \mathrm{s} 41563-018-0262-7$.

(54) Neutzner, S.; Thouin, F.; Cortecchia, D.; Petrozza, A.; Silva, C.; Kandada, A. R. S. Excitonpolaron spectral structures in two-dimensional hybrid lead-halide perovskites. Phys. Rev. Materials 2018, 2, DOI: 10.1103/physrevmaterials.2.064605.

(55) Thouin, F.; Neutzner, S.; Cortecchia, D.; Dragomir, V. A.; Soci, C.; Salim, T.; Lam, Y. M.; Leonelli, R.; Petrozza, A.; Kandada, A. R. S.; Silva, C. Stable biexcitons in two-dimensional metal-halide perovskites with strong dynamic lattice disorder. Phys. Rev. Materials 2018, 2, DOI: $10.1103 /$ physrevmaterials.2.034001.

(56) Sie, E. J. et al. An ultrafast symmetry switch in a Weyl semimetal. Nature 2019, 565, 61-66, DOI: $10.1038 / \mathrm{s} 41586-018-0809-4$.

(57) Morrow, D. J.; Kohler, D. D.; Zhao, Y.; Jin, S.; Wright, J. C. Triple sum frequency pumpprobe spectroscopy of transition metal dichalcogenides. Phys. Rev. B 2019, 100, 235303, DOI: 10.1103/physrevb.100.235303. 


\section{Graphical TOC Entry}

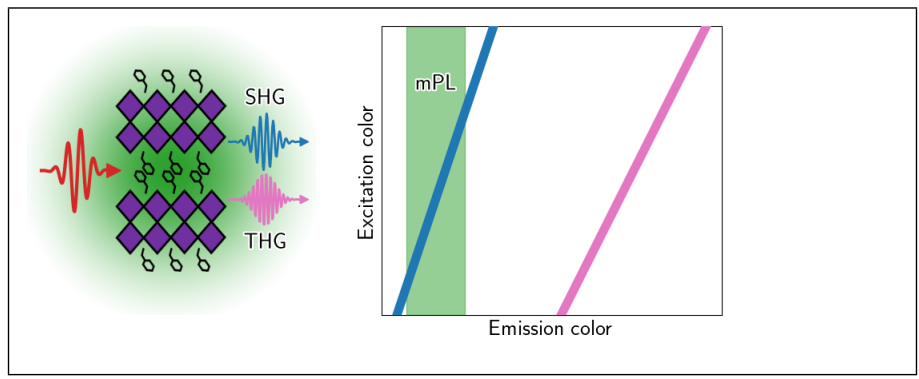

